\title{
Utility of bronchial lavage fluids for epithelial growth factor receptor mutation assay in lung cancer patients: Comparison between cell pellets, cell blocks and matching tissue specimens
}

\author{
SHIHO ASAKA ${ }^{1}$, AKIHIKO YOSHIZAWA ${ }^{1,2}$, RIE NAKATA $^{1}$, TATSUYA NEGISHI ${ }^{1}$, HIROSHI YAMAMOTO ${ }^{3}$, \\ TAKAYUKI SHIINA ${ }^{4}$, SHOHEI SHIGETO ${ }^{1}$, KAZUYUKI MATSUDA ${ }^{1}$, \\ YUKIHIRO KOBAYASHI $^{1}$ and TAKAYUKI HONDA ${ }^{1}$ \\ ${ }^{1}$ Department of Laboratory Medicine, Shinshu University Hospital, Matsumoto, Nagano 390-8621; \\ ${ }^{2}$ Department of Diagnostic Pathology, Kyoto University Hospital, Kyoto 606-8507; ${ }^{3}$ First Department of Internal Medicine, \\ Shinshu University School of Medicine; ${ }^{4}$ Department of Thoracic Surgery, Shinshu University Hospital, \\ Matsumoto, Nagano 390-8621, Japan
}

Received June 7, 2017; Accepted September 1, 2017

DOI: $10.3892 / 01.2017 .7464$

\begin{abstract}
The detection of epidermal growth factor receptor $(E G F R)$ mutations is necessary for the selection of suitable patients with non-small cell lung cancer (NSCLC) for treatment with EGFR tyrosine kinase inhibitors. Cytology specimens are known to be suitable for EGFR mutation detection, although tissue specimens should be prioritized; however, there are limited studies that examine the utility of bronchial lavage fluid (BLF) in mutation detection. The purpose of the present study was to investigate the utility of BLF specimens for the detection of EGFR mutations using a conventional quantitative $E G F R$ polymerase chain reaction (PCR) assay. Initially, quantification cycle $(\mathrm{Cq})$ values of cell pellets, cell-free supernatants and cell blocks obtained from three series of $1 \% E G F R$ mutation-positive lung cancer cell line samples were compared for mutation detection. In addition, PCR analysis of BLF specimens obtained from 77 consecutive NSCLC patients, detecting EGFR mutations was validated, and these results were compared with those for the corresponding formalin-fixed paraffin-embedded (FFPE) tissue specimens obtained by surgical resection or biopsy of
\end{abstract}

Correspondence to: $\operatorname{Dr}$ Akihiko Yoshizawa, Department of Diagnostic Pathology, Kyoto University Hospital, 54 Kawahara, Sakyo, Kyoto 606-8507, Japan

E-mail: akyoshi@kuhp.kyoto-u.ac.jp

Abbreviations: NSCLC, non-small cell lung cancer; EGFR, epidermal growth factor receptor; EGFR-TKIs, EGFR tyrosine kinase inhibitors; BLF, bronchial lavage fluid; FFPE, formalin-fixed paraffin-embedded

Key words: lung cancer, epithelial growth factor receptor, polymerase chain reaction, therascreen, bronchial lavage fluid, cell pellet, cell-free supernatant, FFPE cell block
49 of these patients. The $\mathrm{Cq}$ values for mutation detection were significantly lower in the cell pellet group (average, 29.58) compared with the other groups, followed by those in cell-free supernatants (average, 34.15) and in cell blocks (average, 37.12) for all three series $(\mathrm{P}<0.05)$. Mutational status was successfully analyzed in 77 BLF specimens, and the results obtained were concordant with those of the 49 matching FFPE tissue specimens. Notably, EGFR mutations were even detected in 10 cytological specimens that contained insufficient tumor cells. EGFR mutation testing with BLF specimens is therefore a useful and reliable method, particularly when sufficient cancer cells are not obtained.

\section{Introduction}

Lung cancer is the most common cause of cancer-associated mortality worldwide, accounting for 1.8 million newly diagnosed cases and 1.6 million incidences of cancer-associated mortality in 2012 (1). In recent years, epidermal growth factor receptor tyrosine kinase inhibitors (EGFR-TKIs), including gefitinib, erlotinib or afatinib, have been widely used in the treatment of non-small cell lung cancer (NSCLC) patients, resulting in markedly prolonged progression-free and overall survival times compared with those of patients undergoing standard chemotherapies (2-7). However, the therapeutic effect of EGFR-TKIs is associated with the mutation spectrum and status of EGFR (8). Deletions in exon 19 and L858R in exon 21 are known to sensitize patients to EGFR-TKI therapy $(9,10)$, with these mutations covering $\sim 90 \%$ of oncogenic EGFR mutations (11). By contrast, the T790M substitution in exon 20 is the most common secondary mutation among patients who acquire resistance to EGFR-TKIs (12). Osimertinib, a third-generation TKI that specifically targets the T790M mutation, was introduced to in 2015 and has exhibited clinical efficacy in NSCLC patients with the T790M mutation (13). Thus, in order to select NSCLC patients suitable for EGFR-TKI therapy, it is necessary to perform EGFR molecular testing with cancer specimens from these patients (14). 
In clinical practice, EGFR mutation status is commonly analyzed by polymerase chain reaction (PCR)-based assays using biopsy specimens from advanced NSCLC patients (14); however, cytological specimens are often the only specimens available. Although tissue specimens obtained by transbronchial or transcutaneous biopsies are preferable (14), it is often difficult to obtain sufficient cancer tissue to perform morphological and molecular analyses. Cytological specimens may, however, be useful diagnostic tools for these analyses and the procedures used to obtain these specimens are less invasive than those used to obtain biopsy specimens (15).

Previous studies have documented the use of cytological specimens, including pleural effusion (PLE), for $E G F R$ mutation testing (16-19). However, there are limited studies examining the utility of bronchial lavage fluid (BLF), which is obtained following transbronchial lung biopsy and usually contains fewer cancer cells than PLE (20-22). In addition, data on the reported performance of companion diagnostics, including the therascreen EGFR RGQ PCR kit (the therascreen EGFR assay), primarily rely on formalin-fixed paraffin-embedded (FFPE) tissue blocks from resected or biopsy specimens and as such, there are limited data on fresh cytological specimens (23).

The purpose of the present study was to compare the efficiency of EGFR mutation detection between fresh cytological samples (cell pellets and cell-free supernatants) and FFPE cell blocks prepared from $1 \%$ EGFR mutation-positive lung cancer cell line mixtures using the therascreen EGFR assay. Furthermore, the utility of fresh BLF specimens from patients with NSCLC was also validated against matched FFPE tissue specimens in EGFR mutation detection using the therascreen EGFR assay.

\section{Materials and methods}

Lung cancer cell line samples. A total of three types of cytological samples were prepared: Fresh cell pellets, fresh supernatants, and FFPE cell blocks from three series of lung cancer cell line samples, including $1 \%$ EGFR mutant cells [sample (S) 1,2, and 3]. The following human lung cancer cell lines were used: PC9 [EGFR E746_A750del (c.2235_2249del)] and A549 (EGFR wild-type). The PC9 cell line was obtained directly from the Riken BioResource Centre (Tsukuba, Japan). The A549 cell line was obtained directly from the Japanese Cancer Research Bank (Tokyo, Japan). PC9 and A549 were mixed at a ratio of 1:99, so that the percentage of $E G F R$-mutant cells in the mixture was $1 \%$. The cell line mixture was divided into three samples, S1, S2, and S3, and each sample was washed with saline, centrifuged at $500 \mathrm{xg}$ for $5 \mathrm{~min}$ at room temperature, then divided into cell pellet samples (S1p, S2p, and S3p) and cell-free supernatant samples (S1s, S2s, and S3s). From the cell pellets, FFPE cell block samples (S1b, S2b, and S3b) were produced through fixation with $20 \%$ neutral-buffered formalin for $24 \mathrm{~h}$ at room temperature using a sodium alginate cell block method (Fig. 1).

BLF and matched FFPE tissue specimens from patients with NSCLC. Fresh BLF specimens were collected from 219 patients who were clinically diagnosed with lung cancer between January 2014 and December 2014, and the specimens provided were used for cytological diagnosis at the Department of Laboratory Medicine at Shinshu University Hospital (Matsumoto, Japan). Following cytological evaluation of specimens from 219 patients, specimens that contained normal or benign cells (123 patients), malignant lymphoma (1 patient), renal cell metastatic carcinoma (1 patient), small cell carcinoma (8 patients), and squamous cell carcinoma (9 patients) were all excluded. The remaining 77 patients (age range, 41-85; median age, 69; 54 males and 23 females) whose specimens were cytologically diagnosed as 'primary lung adenocarcinoma', 'NSCLC-not otherwise specified (NSCLC-NOS)', 'suspicious for malignancy' (i.e., suspicious for adenocarcinoma or NSCLC-NOS), or 'atypical cells' meaning indefinite for neoplasia were enrolled in this study. These patients are the same as those used in a previous study by the same authors (22).

EGFR mutation status was analyzed using fresh cell pellets from BLF specimens. Among the 77 patients, 49 patients had EGFR mutation assay results from FFPE tissue specimens obtained by surgical resection or biopsy. The assay results from the BLF specimens were compared with those from the matching FFPE tissue specimens. The present study was reviewed and approved by the Medical Ethical Committee of the Shinshu University School of Medicine. All patients provided written informed consent for inclusion in the present study.

DNA extraction. DNA was extracted from fresh samples (cell pellets and cell-free supernatants) using the QIAamp DNA Blood Mini kit (Qiagen, Inc., Valencia, CA, USA) and the QIAamp DNA FFPE Tissue kit (Qiagen, Inc.) for FFPE specimens, according to the manufacturer's protocol. DNA concentration was quantified by spectrophotometry using a NanoDrop ND100 instrument (Thermo Fisher Scientific, Inc., Waltham, MA, USA). For comparison of the three types of cytological samples using cell lines, the therascreen EGFR assays were performed following adjustment of the DNA concentrations of cell pellets, cell-free supernatants, and FFPE cell blocks to $4.5 \mathrm{ng} / \mu \mathrm{l}$. For the validation study using BLF specimens, the DNA concentrations were adjusted to $<10 \mathrm{ng} / \mu \mathrm{l}$.

EGFR mutation analysis. For EGFR mutation detection, the Rotor-Gene Q 5plex HRM instrument was used with the therascreen EGFR RGQ PCR kit (therascreen EGFR assay; Qiagen, Inc.). This assay is approved for use in the United States, Europe, Japan, and China, and the kit is based on the amplification-refractory mutation system (ARMS) and Scorpion PCR technology, which enable the sensitive and selective site-specific detection of 29 types of somatic mutations in EGFR (24). The reaction conditions were as follows: $95^{\circ} \mathrm{C}$ for $15 \mathrm{~min}$ for $1 \mathrm{cycle} ; 95^{\circ} \mathrm{C}$ for $30 \mathrm{sec}$ and $60^{\circ} \mathrm{C}$ for $60 \mathrm{sec}$ for 40 cycles. The analysis was performed using the Rotor-Gene Q series software, version 2.0.2 (Qiagen, Inc.). For comparison between the three types of cytological samples (cell pellets, cell-free supernatants and FFPE cell blocks) using cell lines, the cycle quantification $(\mathrm{Cq})$ value of the mutant allele in each sample was compared using the therascreen EGFR 'Deletions' assay, which detects EGFR E746_A750del. For the validation study comparing BLF specimens to FFPE tissue specimens, the manufacturer-supplied cut-off delta $\mathrm{Cq}(\Delta \mathrm{Cq})$ values were used to determine the result whether positive or negative for the mutation in each EGFR mutation reaction. 


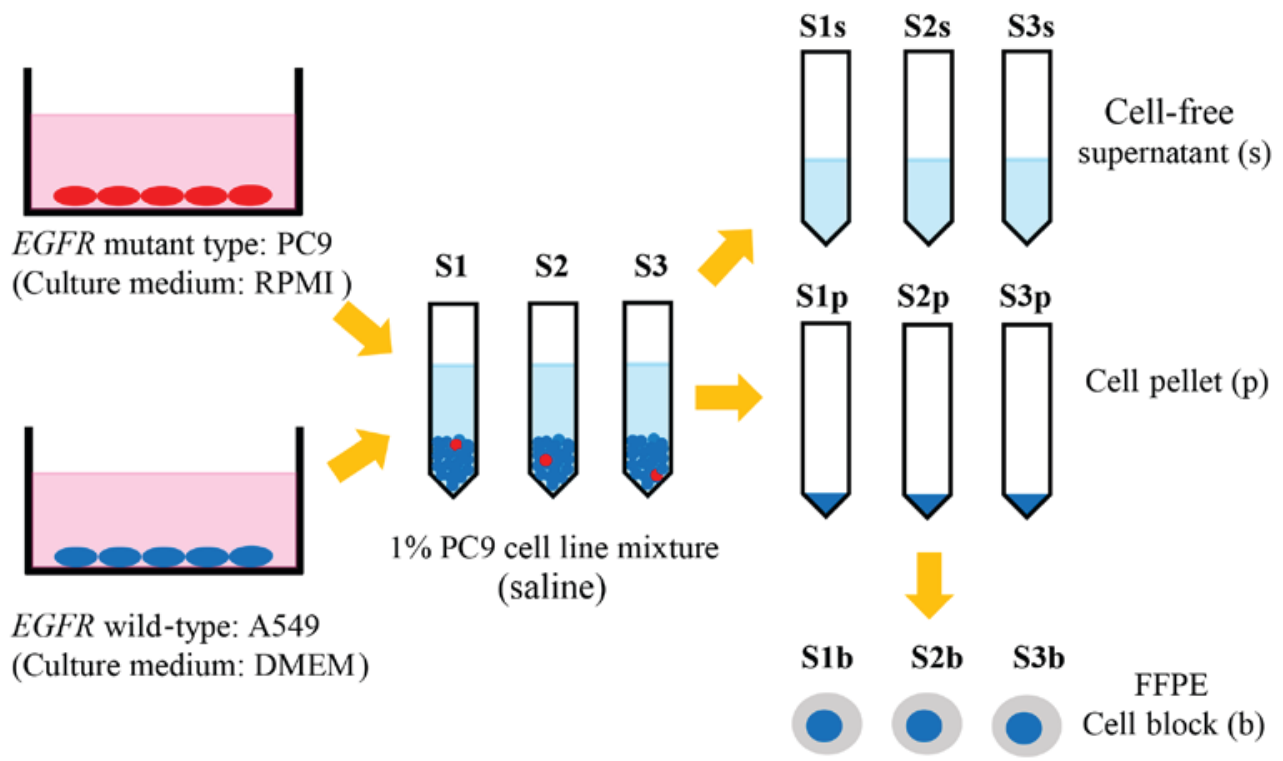

Figure 1. Lung cancer PC9 [EGFR E746_A750del (c.2235_2249del); red ovals] and A549 (EGFR wild-type; blue ovals) cell lines were mixed at a ratio of 1:99, meaning the percentage of EGFR mutant cells in the mixture was $1 \%$. The $1 \%$ EGFR mutation-positive mixture was divided into samples S1, S2 and S3, which were washed with saline, centrifuged and divided into S1p, S2p, and S3p, and S1s, S2s, and S3s. From the cell pellets, S1b, S2b, and S3b were prepared. EGFR, epithelial growth factor receptor; S1, sample 1; S1p, S1 cell pellet; S1s, S1 cell-free supernatant; S1b, S1b formalin-fixed paraffin embedded cell block.

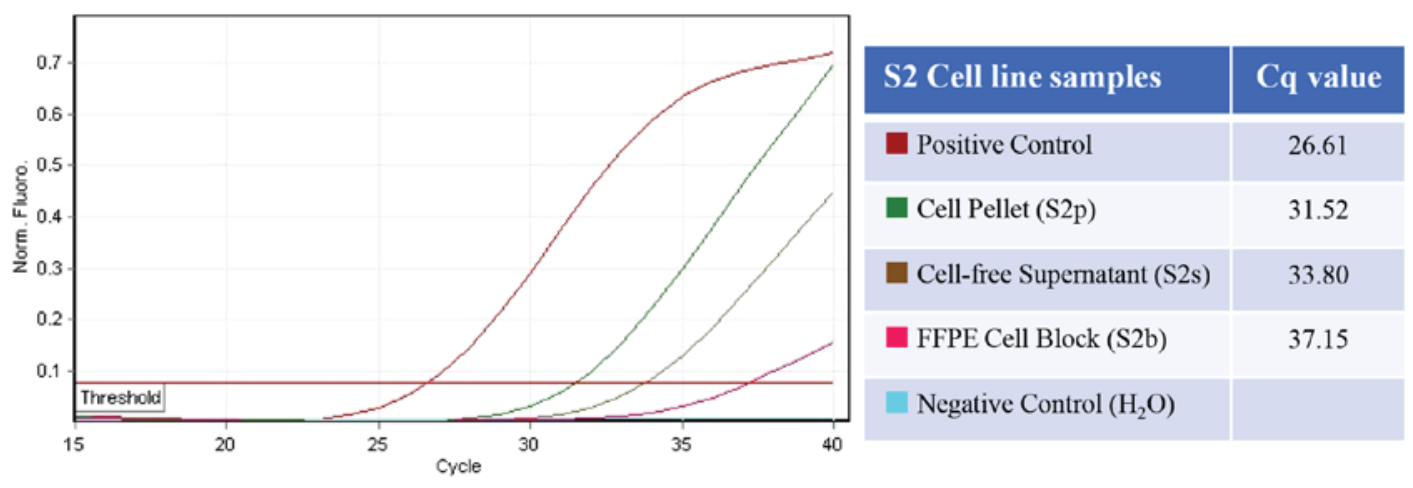

Figure 2. Amplification curves and Cq values of S2p, S2s and S2b, including the manufacturer's positive control and a negative control consisting of nuclease-free water. The lowest $\mathrm{Cq}$ value was observed for the cell pellet, followed by the cell-free supernatant and then the FFPE cellblock. Cq, threshold cycle; FFPE, formalin-fixed paraffin-embedded; S2p, sample 2 cell pellet; S2s, S2 cell-free supernatant; S2b, S2 FFPE cellblock.

Statistical analysis. For comparison of the cell line samples, the Quade test (Statcel version 4; OMS Publishing, Tokorozawa, Japan) was used to compare the differences in Cq values between the cell pellets, cell-free supernatants, and FFPE cell blocks. The Quade test indicates if there is a significant difference in the $\mathrm{Cq}$ values among the three sample types: Cell pellets, cell-free supernatants and FFPE cell blocks. The $\mathrm{Cq}$ values in three series ( $\mathrm{S} 1, \mathrm{~S} 2$ and $\mathrm{S} 3$ ) were compared. $\mathrm{P}<0.05$ was considered to indicate a statistically significant difference.

\section{Results}

Comparison of $\mathrm{Cq}$ values between samples. The $\mathrm{Cq}$ values of the cytological samples in the three series, as determined by the therascreen EGFR assay, are presented in Table I. For all cell line samples excluding the FFPE cell block of S3, the EGFR mutation (E746_A750del) was detected by therascreen EGFR assay within 40 cycles. The average $\mathrm{Cq}$ values for mutation detection for the three sample types in the three series
(S1, S2, and S3) were as follows: 29.58 for fresh cell pellets, 34.15 for fresh cell-free supernatants and 37.12 for FFPE cell blocks. The Quade test revealed that the $\mathrm{Cq}$ values in each series were significantly lower in cell pellets compared with that in the other sample types, followed by cell-free supernatants and FFPE cellblocks $(\mathrm{P}<0.05)$. The representative amplification curve and $\mathrm{Cq}$ value for each sample in the S2 series are presented in Fig. 2.

Assay results of 77 fresh BLF specimens. EGFR mutation status was successfully analyzed using cell pellets from all 77 BLF specimens. The therascreen EGFR assay detected the exon 21 L858R point mutation in 14 patients (18.2\%), exon 19 deletions in 10 patients (12.3\%), and an exon 20 insertion in 1 patient $(1.3 \%)$.

For the 49 patients who had $E G F R$ mutation assay results for matching FFPE tissue specimens, all EGFR assay results were completely concordant between BLF cell pellets and FFPE tissue specimens. The assay results for the BLF cell pellets 
Table I. Cq values of cell line samples in therascreen EGFR assay.

\begin{tabular}{lcccc}
\hline Cq value & $\begin{array}{c}\text { Cell } \\
\text { pellets }\end{array}$ & $\begin{array}{c}\text { Cell-free } \\
\text { supernatants }\end{array}$ & $\begin{array}{c}\text { FFPE } \\
\text { cell blocks }\end{array}$ & P-value \\
\hline S1 & 24.93 & 34.43 & 35.17 & $<0.05$ \\
S2 & 31.52 & 33.80 & 37.15 & \\
S3 & 32.28 & 34.21 & 39.05 & \\
Average & 29.58 & 34.15 & 37.12 & \\
\hline
\end{tabular}

Cq, threshold cycle; S1, sample 1; FFPE, formalin-fixed paraffinembedded.

Table II. EGFR assay results of BLF cell pellets and matching FFPE tissues.

\begin{tabular}{lcc}
\hline $\begin{array}{l}\text { EGFR mutation } \\
\text { status }\end{array}$ & $\begin{array}{c}\text { BLF cell } \\
\text { pellets, n }(\%)\end{array}$ & $\begin{array}{c}\text { Matching FFPE } \\
\text { tissues, n }(\%)\end{array}$ \\
\hline Positive & $17(34.7 \%)$ & $17(34.7 \%)$ \\
Exon 19 deletions & 5 & 5 \\
Exon 21 L858R & 11 & 11 \\
Exon 20 insertions & 1 & 1 \\
Negative & $32(65.3 \%)$ & $32(65.3 \%)$ \\
Total & 49 & 49 \\
\hline
\end{tabular}

$E G F R$, epithelial growth factor receptor; BLF, bronchial lavage fluid; FFPE, formalin-fixed paraffin-embedded.

and matching FFPE tissue specimens in these 49 patients are listed in Table II.

The association between cytological diagnosis and EGFR mutation status of BLF specimens is demonstrated in Table III. EGFR mutations were detected in specimens that were cytologically diagnosed as 'primary lung adenocarcinoma' or 'NSCLC-NOS' from 7 patients, 'suspicious for malignancy' from 6 patients, and 'atypical cells' from 4 patients.

\section{Discussion}

Although the use of FFPE cell block specimens should be prioritized for EGFR mutation testing, various cytological specimens, including liquid-based cytology, smear, fresh cell pellet and supernatant specimens are also suitable (14-16,18,25,26). However, the guidelines of the College of American Pathologists/International Association for the Study of Lung Cancer/Association for Molecular Pathology, currently recommend the creation of FFPE cell blocks from cytological specimens in order to perform morphological and immunohistochemical examination for pathological diagnosis, and to stock them for additional molecular diagnostic studies (14). A novel EGFR mutation assay (the EGFR d-PCR assay) using fresh liquid cytological specimens (cell pellets) was previously validated, revealing that these specimens may contribute to the rapid and simple point-of-care test (22). In
Table III. EGFR mutation status and cytological diagnosis of 49 BLF specimens.

\begin{tabular}{lccr}
\hline Cytological & $\begin{array}{c}\text { EGFR- } \\
\text { mutation } \\
\text { diagnosis }\end{array}$ & $\begin{array}{c}\text { EGFR- } \\
\text { mutation }\end{array}$ & \\
\hline ADC or NSCLC-NOS & 7 & 27 & 34 \\
Suspicious for malignancy & 6 & 3 & 9 \\
Atypical cells & 4 & 2 & 6 \\
Total & 17 & 32 & 49 \\
\hline
\end{tabular}

$E G F R$, epithelial growth factor receptor; BLF, bronchial lavage fluid, ADC, adenocarcinoma; NSCLC-NOS, non-small cell lung cancer, not otherwise specified.

the present study, the detection efficiency for fresh cytological samples (not only cell pellets but also cell-free supernatants) was demonstrated, as was the relative EGFR mutation detection accuracy for fresh cell pellets from BLF specimens versus FFPE tissue specimens.

When comparing cell pellets, cell-free supernatants and FFPE cell blocks from cancer cell lines in the present study, cell pellets had significantly lower $\mathrm{Cq}$ values compared with FFPE cell blocks. This is because FFPE samples suffer from DNA fragmentation and the formation of cross-links during the fixation process (27); therefore, fresh cell pellets are considered to contain DNA that is more efficacious for mutation detection compared with FFPE cell blocks. In addition, DNA derived from slices of FFPE cell blocks usually contain DNA from only a small number of cancer cells, whereas fresh cytological specimens contain DNA from a number of cancer cells (28). Hence, FFPE cell block samples are assumed to exhibit a higher risk of false-negative outcomes compared with fresh cell pellets from cytological specimens $(29,30)$.

In the present study, cell-free supernatant samples, which are considered to contain less DNA than fresh cell pellets, exhibited significantly better amplification efficiency compared with FFPE cell blocks. The result from the present study indicated that the fixation process has a strong negative impact on the detection efficiency of EGFR mutations and is indicative of the use of fresh cell-free supernatants of cytological specimens for EGFR mutation detection assays. Previous studies have reported the feasibility of detecting EGFR mutations with fresh cell-free supernatants of PLEs by direct sequencing (31), mutant-enriched PCR (19) and Scorpion ARMS (17). Zhang et al (19) revealed a good concordance between $E G F R$ mutation assay results from cell pellets and those from matching cell-free fluids following mutant-enriched PCR. By using highly sensitive methods for $E G F R$ mutation detection, performing EGFR assays with cell-free supernatants of BLF specimens is possible and may be useful, as the cell pellets, which enable morphological and multiple molecular analyses are preserved.

In the validation of the EGFR mutational status of BLF specimens using cell pellets from 77 patients with NSCLC, all EGFR mutational statuses were successfully analyzed and there were no specimens that failed to amplify. The assay results for 
all 49 BLF cell pellets with matching FFPE tissue specimens were completely concordant between the two specimen types. Goto et al (16) reported an excellent interassay concordance between 29 bronchofiberscopic brushing cytological specimens and matching FFPE specimens using Scorpion ARMS, PCR-Invader, PNA-LNA PCR clamp, and Cycleave PCR, with concordance rates of 93.1-96.6\% (к-coefficients, 0.86-0.93). Khode et al (32) compared 37 paired cytological smears with matched FFPE surgical tissue specimens from the same anatomical sites using pyrosequencing and RT-PCR platforms, and additionally reported a concordance rate of $97 \%$ between the two sample types. The present study also demonstrated the accuracy of the EGFR assay using fresh cytological specimens for $E G F R$ mutation detection, with high concordance following the therascreen EGFR assay results of FFPE tissue specimens.

Among the 77 fresh BLF specimens, EGFR mutations were even detected in specimens containing only a few cancer cells, which were cytologically diagnosed as 'atypical cells' (i.e., indefinite for neoplasia). As was demonstrated by comparing different cytological samples from cell lines, use of the EGFR mutation assay with fresh cell pellets resulted in higher sensitivity of mutation detection compared with the use of FFPE cell blocks, even with a small amount of mutant DNA derived from a few cancer cells being detected by the assay. Furthermore, the use of BLF specimens may also markedly reduce the time and effort required for DNA extraction compared with the use of FFPE specimens, as BLF specimens do not require deparaffinization or reversal of formaldehyde-induced nucleic acid modification. Thus, the EGFR mutation assay with fresh BLF specimens may represent a screening method for diagnosing whether atypical cells are cancerous or not, particularly when morphological diagnosis is difficult owing to the presence of insufficient material.

Despite the fact that fresh BLF specimens contain DNA that is more efficacious for an EGFR mutation detection assay, they also usually contain more non-cancerous cells, including inflammatory cells and benign epithelial cells, compared with tissue specimens. The amount of DNA from non-cancerous cells affects the quality of the mutation-specific PCR assay, which may lead to false-negative results. Furthermore, for the current major companion diagnostics for EGFR-TKIs, including the therascreen $E G F R$ assay, the cut-off values have been determined for DNA samples extracted from FFPE tissue or cell block specimens, as fresh cytological specimens are not generally used for testing. Therefore, the results of testing fresh cytological specimens should be interpreted carefully, and priority should be given to FFPE tissue specimens or cell blocks if they contain sufficient cancer cells.

To conclude, EGFR mutation detection assays using fresh BLF specimens offer a sensitive, accurate, simple and time-saving method for detection of EGFR mutations, even when sufficient cancer tissues or cytological specimens are not obtained. This method enables the full use of specimens from NSCLC patients for multiple molecular analyses.

\section{Acknowledgements}

The present study was partly supported by the Japan Society for the Promotion of Science Grants-in-Aid for Scientific Research, commonly termed KAKENHI (grant no. 25460434).

\section{References}

1. Torre LA, Bray F, Siegel RL, Ferlay J, Lortet-Tieulent J and Jemal A: Global Cancer Statistics, 2012. CA Cancer J Clin 65: 87-108, 2015.

2. Maemondo M, Inoue A, Kobayashi K, Sugawara S, Oizumi S, Isobe $\mathrm{H}$, Gemma A, Harada M, Yoshizawa H, Kinoshita I, et al: Gefitinib or chemotherapy for non-small-cell lung cancer with mutated EGFR. N Engl J Med 362: 2380-2388, 2010.

3. Mitsudomi T, Morita S, Yatabe Y, Negoro S, Okamoto I, Tsurutani J, Seto T, Satouchi M, Tada H, Hirashima T, et al: Gefitinib versus cisplatin plus docetaxel in patients with non-small-cell lung cancer harbouring mutations of the epidermal growth factor receptor (WJTOG3405): An open label, randomised phase 3 trial. Lancet Oncol 11: 121-128, 2010.

4. Zhou C, Wu YL, Chen G, Feng J,Liu XQ, Wang C, Zhang S, Wang J, Zhou S, Ren S, et al: Erlotinib versus chemotherapy as first-line treatment for patients with advanced EGFR mutation-positive non-small-cell lung cancer (OPTIMAL, CTONG-0802): A multicentre, open-label, randomised, phase 3 study. Lancet Oncol 12: 735-742, 2011.

5. Rosell R, Carcereny E, Gervais R, Vergnenegre A, Massuti B, Felip E, Palmero R, Garcia-Gomez R, Pallares C, Sanchez JM, et al: Erlotinib versus standard chemotherapy as first-line treatment for European patients with advanced EGFR mutation-positive non-small-cell lung cancer (EURTAC): A multicentre, open-label, randomised phase 3 trial. Lancet Oncol 13: 239-246, 2012

6. Wu YL, Zhou C, Hu CP, Feng J, Lu S, Huang Y, Li W, Hou M, Shi JH, Lee KY, et al: Afatinib versus cisplatin plus gemcitabine for first-line treatment of Asian patients with advanced non-small-cell lung cancer harbouring EGFR mutations (LUX-Lung 6): An open-label, randomised phase 3 trial. Lancet Oncol 15: 213-222, 2014.

7. Yang JC, Wu YL, Schuler M, Sebastian M, Popat S, Yamamoto N, Zhou C, Hu CP, O'Byrne K, Feng J, et al: Afatinib versus cisplatin-based chemotherapy for EGFR mutation-positive lung adenocarcinoma (LUX-Lung 3 and LUX-Lung 6): Analysis of overall survival data from two randomised, phase 3 trials. Lancet Oncol 16: 141-151, 2015.

8. Fukuoka M, Wu YL, Thongprasert S, Sunpaweravong P, Leong SS, Sriuranpong V, Chao TY, Nakagawa K, Chu DT, Saijo N, et al: Biomarker analyses and final overall survival results from a phase III, randomized, open-label, first-line study of gefitinib versus carboplatin/paclitaxel in clinically selected patients with advanced non-small-cell lung cancer in Asia (IPASS). J Clin Oncol 29: 2866-2874, 2011.

9. Naidoo J, Sima CS, Rodriguez K, Busby N, Nafa K, Ladanyi M, Riely GJ, Kris MG, Arcila ME and Yu HA: Epidermal growth factor receptor exon 20 insertions in advanced lung adenocarcinomas: Clinical outcomes and response to erlotinib. Cancer 121: 3212-3220, 2015.

10. Lee VH, Tin VP, Choy TS, Lam KO, Choi CW, Chung LP, Tsang JW, Ho PP, Leung DK, Ma ES, et al: Association of Exon 19 and 21 EGFR mutation patterns with treatment outcome after first-line tyrosine kinase inhibitor in metastatic non-small-cell lung cancer. J Thorac Oncol 8: 1148-1155, 2013.

11. Mitsudomi T, Kosaka T and Yatabe Y: Biological and clinical implications of EGFR mutations in lung cancer. Int J Clin Oncol 11: 190-198, 2006.

12. Kuiper JL, Heideman DA, Thunnissen E, Paul MA, van Wijk AW, Postmus PE and Smit EF: Incidence of T790M mutation in (sequential) rebiopsies in EGFR-mutated NSCLC-patients. Lung Cancer 85: 19-24, 2014.

13. Wang S, Cang S and Liu D: Third-generation inhibitors targeting EGFR T790M mutation in advanced non-small cell lung cancer. J Hematol Oncol 9: 34, 2016.

14. Lindeman NI, Cagle PT, Beasley MB, Chitale DA, Dacic S, Giaccone G, Jenkins RB, Kwiatkowski DJ, Saldivar JS, Squire J, et al: Molecular testing guideline for selection of lung cancer patients for EGFR and ALK tyrosine kinase inhibitors guideline from the College of American Pathologists, International Association for the Study of Lung Cancer, and Association for Molecular Pathology. J Mol Diagn 15: 415-453, 2013.

15. Ellison G, Zhu GS, Moulis A, Dearden S, Speake G and McCormack R: EGFR mutation testing in lung cancer: A review of available methods and their use for analysis of tumour tissue and cytology samples. J Clin Pathol 66: 79-89, 2013.

16. Goto K, Satouchi M, Ishii G, Nishio K, Hagiwara K, Mitsudomi T, Whiteley J, Donald E, McCormack R and Todo T: An evaluation study of EGFR mutation tests utilized for non-small-cell lung cancer in the diagnostic setting. Ann Oncol 23: 2914-2919, 2012. 
17. Kimura H, Fujiwara Y, Sone T, Kunitoh H, Tamura T, Kasahara K and Nishio K: High sensitivity detection of epidermal growth factor receptor mutations in the pleural effusion of non-small cell lung cancer patients. Cancer Sci 97: 642-648, 2006.

18. Lin J, Gu Y, Du R, Deng M, Lu Y and Ding Y: Detection of EGFR mutation in supernatant, cell pellets of pleural effusion and tumor tissues from non-small cell lung cancer patients by high resolution melting analysis and sequencing. Int J Clin Exp Pathol 7: 8813-8822, 2014.

19. Zhang X, Zhao Y, Wang M, Yap WS and Chang AY: Detection and comparison of epidermal growth factor receptor mutations in cells and fluid of malignant pleural effusion in non-small cell lung cancer. Lung Cancer 60: 175-182, 2008.

20. Yamaguchi F, Kugawa S, Tateno H, Kokubu F and Fukuchi K: Analysis of EGFR, KRAS and P53 mutations in lung cancer using cells in the curette lavage fluid obtained by bronchoscopy. Lung Cancer 78: 201-206, 2012.

21. Sakamoto T, Kodani M, Takata M, Chikumi H, Nakamoto M, Nishii-Ito S, Ueda Y, Izumi H, Makino H, Touge H, et al: A novel point-of-care system for high-speed real-time polymerase chain reaction testing for epidermal growth factor receptor mutations in bronchial lavage fluids after transbronchial biopsy in patients with non-small cell lung cancer. Int J Oncol 46: 1473-1480, 2015.

22. Asaka S, Yoshizawa A, Matsuda K, Yamaguchi A, Yamamoto H, Shiina T, Nakata R, Ogawa K, Zhang $M$ and Honda T: A novel, rapid point-of-care test for lung cancer patients to detect epidermal growth factor receptor gene mutations by using real-time droplet-PCR and fresh liquid cytology specimens. Oncol Rep 37: 1020-1026, 2017.

23. Syed YY: Therascreen ${ }^{\circledR}$ EGFR RGQ PCR Kit: A companion diagnostic for afatinib and gefitinib in non-small cell lung cancer. Mol Diagn Ther 20: 191-198, 2016.

24. Vallée A, Le Loupp AG and Denis MG: Efficiency of the Therascreen ${ }^{\circledR}$ RGQ PCR kit for the detection of EGFR mutations in non-small cell lung carcinomas. Clin Chim Acta 429: 8-11, 2014

25. Lozano MD, Zulueta JJ, Echeveste JI, Gúrpide A, Seijo LM, Martín-Algarra S, Del Barrio A, Pio R, Idoate MA, Labiano T and Perez-Gracia JL: Assessment of epidermal growth factor receptor and K-ras mutation status in cytological stained smears of non-small cell lung cancer patients: Correlation with clinical outcomes. Oncologist 16: 877-885, 2011.
26. Reynolds JP, Tubbs RR, Minca EC, MacNamara S, Almeida FA, Ma PC, Pennell NA and Cicenia JC: EGFR mutational genotyping of liquid based cytology samples obtained via fine needle aspiration (FNA) at endobronchial ultrasound of non-small cell lung cancer (NSCLC). Lung Cancer 86: 158-163, 2014.

27. Dedhia P, Tarale S, Dhongde G, Khadapkar R and Das B: Evaluation of DNA extraction methods and real time PCR optimization on formalin-fixed paraffin-embedded tissues. Asian Pac J Cancer Prev 8: 55-59, 2007.

28. Gailey MP, Stence AA, Jensen CS and Ma DQ: Multiplatform comparison of molecular oncology tests performed on cytology specimens and formalin-fixed, paraffin-embedded tissue. Cancer Cytopathol 123: 30-39, 2015.

29. Harada S, Agosto-Arroyo E, Levesque JA, Alston E, Janowski KM, Coshatt GM and Eltoum IA: Poor cell block adequacy rate for molecular testing improved with the addition of Diff-Quik-stained smears: Need for better cell block processing. Cancer Cytopathol 123: 480-487, 2015.

30. Xu H, Sun W, Zhang G and Cheng Y: Detection of epidermal growth factor receptor mutation in non-small-cell lung carcinoma using cytological and histological specimens. J BUON 20: $142-145,2015$.

31. Kimura H, Fujiwara Y, Sone T, Kunitoh H, Tamura T, Kasahara K and Nishio K: EGFR mutation status in tumour-derived DNA from pleural effusion fluid is a practical basis for predicting the response to gefitinib. Br J Cancer 95: 1390-1395, 2006.

32. Khode R, Larsen DA, Culbreath BC, Parrish S, Walker KL, Sayage-Rabie L, Beissner RS and Rao A: Comparative study of epidermal growth factor receptor mutation analysis on cytology smears and surgical pathology specimens from primary and metastatic lung carcinomas. Cancer Cytopathol 121: 361-369, 2013.

This work is licensed under a Creative Commons Attribution-NonCommercial-NoDerivatives 4.0 International (CC BY-NC-ND 4.0) License. 